\title{
Assessment of the TCA functional in computational chemistry and solid-state physics
}

\author{
E. Fabiano and F. Della Sala \\ Istituto Nanoscienze-CNR, Euromediterranean Center for Nanomaterial \\ Modelling and Technology (ECMT), via Arnesano 73100, Lecce and \\ Center for Biomolecular Nanotechnologies@UNILE, \\ Istituto Italiano di Tecnologia, Via Barsanti, I-73010 Arnesano, Italy \\ L. A. Constantin \\ Center for Biomolecular Nanotechnologies@UNILE, \\ Istituto Italiano di Tecnologia, Via Barsanti, I-73010 Arnesano, Italy \\ A. Terentjevs \\ Istituto Nanoscienze-CNR, Euromediterranean Center for Nanomaterial \\ Modelling and Technology (ECMT), via Arnesano 73100, Lecce \\ P. Cortona \\ Laboratoire Structures, Propriétés et Modélisation des Solides, \\ Université Paris-Saclay, CentraleSupélec, CNRS UMR 8580, \\ Grande Voie des Vignes, F-92295 Châtenay-Malabry, France
}

(Dated: August 30, 2018)

\begin{abstract}
We assess the Tognetti-Cortona-Adamo (TCA) generalized gradient approximation correlation functional [J. Chem. Phys. 128:034101 (2008)] for a variety of electronic systems. We find that, even if the TCA functional is not exact for the uniform electron gas, it is very accurate for the jellium surface correlation energies and it gives a realistic description of the quantum oscillations and surface effects of various jellium clusters, that are important model systems in computational chemistry and solid-state physics. When the TCA correlation is combined with the non-empirical $\mathrm{PBEint}, \mathrm{Wu}-\mathrm{Cohen}$, and $\mathrm{PBEsol}_{b}$ exchange functionals, the resulting exchange-correlation approximations provide good performances for a broad palette of systems and properties, being reasonably accurate for thermochemistry and geometry of molecules, transition metal complexes, non-covalent interactions, equilibrium lattice constants, bulk moduli, and cohesive energies of solids.
\end{abstract}

\section{INTRODUCTION}

Most ab initio electronic structure calculations are nowadays performed in the framework of Kohn-Sham density functional theory (DFT) [1, 2]. The key ingredient of a DFT calculation is the so-called exchangecorrelation (XC) functional, which is the only element of the theory that must be approximated. Thus, the search for efficient XC functionals is a topic of high interest.

There is a great variety of XC approximations. They are usually classified on the so called Jacob's ladder of DFT [3], which includes several families of functionals: local [4 6 , semilocal [7 26], hybrid [27 33$]$ and fully nonlocal 34 37 functionals. Each one these families has its own advantages and limitations in terms of accuracy and computational cost. Hence, no "best" or "universal" functional actually exists in practice. Even the simple semilocal functionals display good utility in many situations. This is especially true for the generalized gradient approximation (GGA) functionals that are often the methods of choice when large systems are considered. For example, in biochemical applications or in solid-state physics, high-level approaches can hardly be applied, due to an overwhelming computational cost; instead, quite reliable results can be obtained at the semilocal level of theory. Thus, the search for GGAs having broad appli- cability is an active research field in DFT [32, 38-44].

To date, numerous GGAs have been considered in literature. Most of them were constructed as whole XC approximations, to benefit of error compensation between the exchange and correlation contributions. These are indeed quite relevant at the semilocal level of theory. Nevertheless, some GGA functionals were also devised as exchange- or correlation-only approximations. In such cases, the correct association of the exchange functional with the correlation one is an important issue to allow a practical use of the functional.

Among the GGA correlation functionals, few variants have gained good popularity. In particular, PBE correlation [8] has been often used as a prototype for nonempirical GGA correlation. Similarly famous is the LeeYang-Parr (LYP) correlation functional [15], obtained as a semilocal approximation of the Colle-Salvetti 45] formula. More recently, correlation functionals based on the uniform electron gas with a gap have been also proposed [16, 46]. Finally, Tognetti, Cortona, and Adamo built a GGA functional, called TCA [17], based on the local Ragot-Cortona correlation [6] and an average reducedgradient analysis.

A detailed analysis of the TCA functional is the aim of this paper. This correlation functional has been the object of only few studies in the past 17, 47 53]. In 
particular, no systematic study of the possibility of combining the TCA correlation functional with one of the existing exchange functionals has been performed. Previous studies just showed that using the TCA correlation together with the PBE exchange [8] yields good results for molecules [17, 48 50] but slightly worsens the performances for solids (with repsect to $\mathrm{PBE}$ ) [51]. On the other hand, using the TCA correlation functional together with the PBEsol exchange [10] some improvement has been achieved for solid-state properties (especially for cohesive energies), but molecular properties have been observed to be quite poorly described.

In this paper, we consider these issues and we analyze in detail the behavior of the TCA correlation for different properties and systems, including jellium spheres and semi-infinite jellium surfaces. Furthermore, we assess the possibility to couple the TCA correlation with some popular GGA exchange functionals. These tests are conducted over a fairly large set of molecular and solid-state properties and systems relevant for semilocal functionals. Hence, a comprehensive understanding of the possible performance of the TCA correlation and its associated XC functionals can be obtained.

\section{METHODOLOGY}

To assess the TCA correlation functional we considered its performance both as a correlation-only functional and in conjuction with different exchange GGA functionals.

For the former task we performed a series of tests, analyzing the ability of TCA to reproduce the correlation energies in different systems. In particular, we calculated the correlation energies of jellium clusters and semiinfinite jellium surface energies. For these calculations we employed accurate LDA Kohn-Sham densities [54]. Moreover, we computed the correlation energy of several atoms and molecules using Hartree-Fock densities and a cc-pV5Z basis set 55 58.

To assess the TCA correlation in conjunction with GGA exchange, we considered a family of PBE-like exchange functionals, which includes PBE [8], PBEsol [10], and PBEint [11]. We also tested the TCA correlation in association with several popular GGA exchange functionals, namely B88 [14], OPTX [59], Wu-Cohen [20], and a recently introduced variant of the $\mathrm{PBEsol}$ exchange (here denoted $\mathrm{PBEsol}_{b}$ ) [60]. The corresponding $\mathrm{XC}$ functionals are denoted PBE-TCA, SOL-TCA, INT-TCA, BTCA, O-TCA, WC-TCA, and $\mathrm{SOL}_{b}$-TCA, respectively.

To perform this assessment we employed a large database covering most of the problems of interest in quantum chemistry and solid-state physics that can be described reasonably well at the semilocal level of theory. For this reason we do not consider tests that require higher level treatments, such as dispersion interactions, dipole moments, and absolute energies. In detail our test suite can be summarized as follows:

- Main group thermochemistry: atomization en- ergies of small molecules (AE6 [61, 62], W4 63 65], G2/97 66, 67]), barrier heights (BH76 64, 65, 68, 69]), reaction energies (BH76RC 64, 65, 68, 69], OMRE [32]), and both barrier heights and reaction energies (K9 [62, 70]).

- Main group geometry: bond lengths of hydrogenic (MGHBL9 [1]) and non-hydrogenic (MGNHBL11 71]) bonds as well as vibrational frequencies (F38 [72]) of small organic molecules.

- Transition metals: atomization energies of small transition metal complexes (TM10AE [12, 73]) and gold clusters (AUnAE [12, 75], reaction energies of transition metal complexes (TMRE [32, 73]), and bond lengths of transition metal complexes (TMBL [12, 74]) and gold clusters (AuBL6 [32, 75]).

- Non-covalent interactions: interaction energies of hydrogen-bond (HB6 [76]), dipole-dipole (DI6 76]), and dihydrogen-bond complexes (DHB23 77]).

- Other molecular properties: difficult cases for DFT (DC9/12 [78]), small gold-organic interfaces (SI12 [32]), and atomization energies of molecules with non-single-reference character (W4-MR [63])

- Solid-state: Equilibrium lattice constants (LC29), bulk moduli (BM29), and cohesive energies (CE29) of 29 solids, including $\mathrm{Al}, \mathrm{Ca}, \mathrm{K}, \mathrm{Li}, \mathrm{Na}, \mathrm{Sr}, \mathrm{Ba}$ (simple metals); Ag, Cu, Pd, Rh, V, Pt, Ni (transition metals); LiCl, $\mathrm{LiF}, \mathrm{MgO}, \mathrm{NaCl}, \mathrm{NaF}$ (ionic solids); AlN, BN, BP, C (insulators); GaAs, GaP, $\mathrm{GaN}, \mathrm{Si}, \mathrm{SiC}, \mathrm{Ge}$ (semiconductors). Reference data to construct this set were taken from Refs. [79, 80].

All calculations concerning this test suite, except solidstate ones, have been performed with the TURBOMOLE program package [81] using the def2-TZVPP basis set [82, 83] and standard molecular integration grids (gridsize 3 option in TURBOMOLE). For transition metal atoms, scalar relativistic effective core potentials (ECP) [84, 85] have been employed. Note that reference data of all tests are corrected for thermal effects, thus they are directly comparable with the outcome of the calculations. In the case of gold clusters, also other relativistic effects beyond the ECP treatment are included in the correction 75]. Fixed reference geometries have been employed in all molecular calculations.

Solid-state calculations have been performed with the VASP program [86] using PBE-PAW pseudopotentials. Note that the use of PAW core potentials ensures good transferability for multiple functionals [80], since the core-valence interaction is recalculated for each functional. Indeed, test calculations employing different PAW potentials have shown that the convergence level of our results is about $1 \mathrm{~m} \AA$ for lattice constants, $0.5 \mathrm{GPa}$ for bulk moduli, and $0.01 \mathrm{eV}$ for cohesive energies. All Brillouin zone integrations were performed on $\Gamma$-centered 
symmetry-reduced Monkhorst-Pack $k$-point meshes, using the tetrahedron method with Blöch corrections. For all the calculations a $24 \times 24 \times 24 k$-mesh grid was used and the plane-wave cutoff was chosen to be $30 \%$ larger than the maximum cutoff for the pseudopotentials of the considered atoms. The bulk modulus was obtained using the Murnaghan equation of state. The cohesive energy, defined as the energy per atom needed to atomize the crystal, was calculated as minus the difference between the total energy of the crystal at its equilibrium volume and the sum of the energies of the constituent atoms as obtained from spin-polarized symmetry-broken calculations. To generate symmetry breaking solutions, atoms were placed in a large orthorhombic box with dimensions $13 \times 14 \times 15 \AA^{3}$. All reference data in the solid-state database are corrected for zero-point phonon effects.

Calculations for the two example applications, reported at the end of the paper (see Fig. [6), were performed using data and computational setups of Refs. [11, 87].

To evaluate the overall performance of each functional, as well as its accuracy for different classes of problems, each class being represented by a set of tests as described above, we considered the mean logarithmic relative absolute error (LRAE), defined in the following way. Let $\langle\mathrm{MAE}\rangle_{i}$ be the average of the mean absolute errors (MAE) of the considered functionals for the test $i$ (in this work we excluded from the average the best and the worst functionals). The LRAE of a given functional is then defined as

$\operatorname{LRAE}[$ functional $]=\frac{100}{M} \sum_{i=1}^{M} \log _{10}\left(\frac{\mathrm{MAE}_{i}[\text { functional }]}{\langle\mathrm{MAE}\rangle_{i}}\right)$

where $M$ is the number of the considered tests. This quantity actually summarizes the performances of the functional for a heterogeneous set of data, since it treats adimensional quantities. Note also that the use of the logarithm makes it independent (up to a rigid shift) from the $\langle\mathrm{MAE}\rangle_{i}$ values, in the sense that, changing the definition $\langle\mathrm{MAE}\rangle_{i}$ (e.g including other functionals in the benchmark), the LRAE of each functional will change, but the differences between the LRAEs will be unchanged (thus, the ordering will not change). Hence, more negative values of LRAE indicate that a functional is performing better than the average of all the considered functionals; oppositely, positive values of the LRAE indicate a performance worse than the average.

\section{A. TCA correlation}

The TCA correlation energy functional [17] is defined as

$$
\begin{aligned}
E_{c}^{T C A}= & \int n \epsilon_{c}^{T C A}\left(r_{s}, \zeta, s\right) d \mathbf{r}= \\
& =\int \frac{3}{4 \pi r_{s}^{3}} \epsilon_{c}^{R C}\left(r_{s}(\mathbf{r})\right) B(s(\mathbf{r})) C(\zeta(\mathbf{r})) d \mathbf{r}
\end{aligned}
$$

where $r_{s}=(3 /[4 \pi n])^{1 / 3}$, with $n$ being the total electron density,

$$
\begin{aligned}
\epsilon_{c}^{R C}\left(r_{s}\right)= & \frac{-0.655868 \arctan \left(4.888270+3.177037 r_{s}\right)}{r_{s}}+ \\
& +\frac{0.897889}{r_{s}}
\end{aligned}
$$

is the local Ragot-Cortona correlation energy per particle [6],

$$
B(s)=\frac{1}{1+\sigma s^{\alpha}},
$$

with $\sigma=1.41$ and $\alpha=2.3$, is an enhancement factor depending on the reduced gradient $s=$ $|\nabla n| /\left[2\left(3 \pi^{2}\right)^{1 / 3} n^{4 / 3}\right]$, and

$$
C(\zeta)=\frac{\left[(1+\zeta)^{2 / 3}+(1-\zeta)^{2 / 3}\right]^{3}}{2^{3}}
$$

is a spin factor (see also Ref. 88]), with $\zeta=\left(n_{\uparrow}-\right.$ $\left.n_{\downarrow}\right) / n\left(n_{\uparrow}\right.$ and $n_{\downarrow}$ are the spin-up and spin-down electron densities, respectively).

In the slowly-varying density limit, for the spinunpolarized case, the TCA correlation has the gradient expansion

$$
\epsilon_{c}^{T C A} \approx \epsilon_{c}^{R C}-\sigma \epsilon_{c}^{R C} s^{\alpha} .
$$

This is formally different from the exact one [8, 22]

$$
\epsilon_{c} \approx \epsilon_{c}^{L D A}+\beta\left(r_{s}\right) t^{2}
$$

where $\epsilon_{c}^{L D A}$ is the correlation energy per particle in the local density approximation (LDA), $\beta$ is the second-order gradient expansion coefficient, and $t=|\nabla n| / 2 k_{s} \phi n$ is the reduced gradient for correlation [7], with $k_{s}=\left(4 k_{F} / \pi\right)^{1 / 2}$ being the Thomas-Fermi screening wave vector $\left(k_{F}=\right.$ $\left.\left(3 \pi^{2} n\right)^{1 / 3}\right)$, and $\phi=\sqrt[3]{C}$ being a spin-scaling factor. Nevertheless, as shown in Fig. 11, the slowly-varying behavior of the TCA correlation functional is similar to the exact one over a quite large range of values of $r_{s}$ and $s$. Thus, the TCA correlation can be expected to work fairly well for systems with a slowly varying density. On the other hand, because of the presence of the exponent $\alpha$ in Eq. (6), when the TCA correlation is used in conjunction with an exchange functional having a gradient expansion of the form $\epsilon_{x} \approx \epsilon_{x}^{L D A}\left(1+\mu s^{2}\right)$, i.e. satisfying the secondorder gradient expansion or any of its modifications, it is not possible to enforce exactly the accurate LDA linear response behavior for the whole resulting XC functional. We recall that this constraint was instead used in the construction of several GGA functionals [8, 33, 89, 90].

In Fig. 2, we show the correlation enhancement factor

$$
F_{c}\left(r_{s}, s, \zeta\right)=\epsilon_{c}\left(n_{\uparrow}, n_{\downarrow}, \nabla n_{\uparrow}, \nabla n_{\downarrow}\right) / \epsilon_{x}^{L D A}(n),
$$

versus the reduced gradient $s$, for $r_{s}=2$, and $\zeta=0$. At $s=0$, TCA recovers the RC local correlation, PBE 


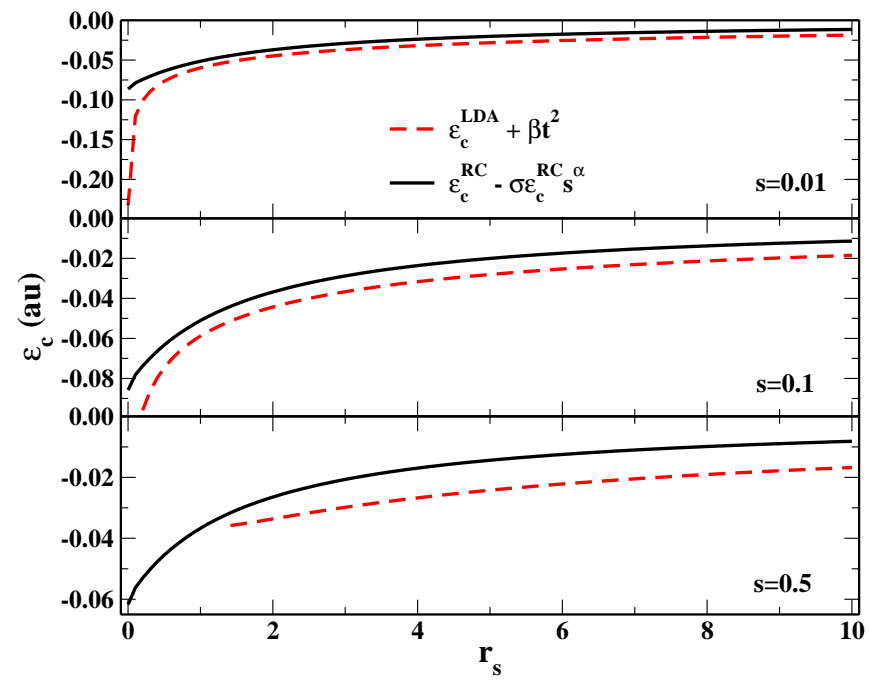

FIG. 1: Gradient expansions of the exact correlation and the TCA one for different values of the reduced gradient $s=$ $|\nabla n| /\left[2\left(3 \pi^{2}\right)^{1 / 3} n^{4 / 3}\right]$.

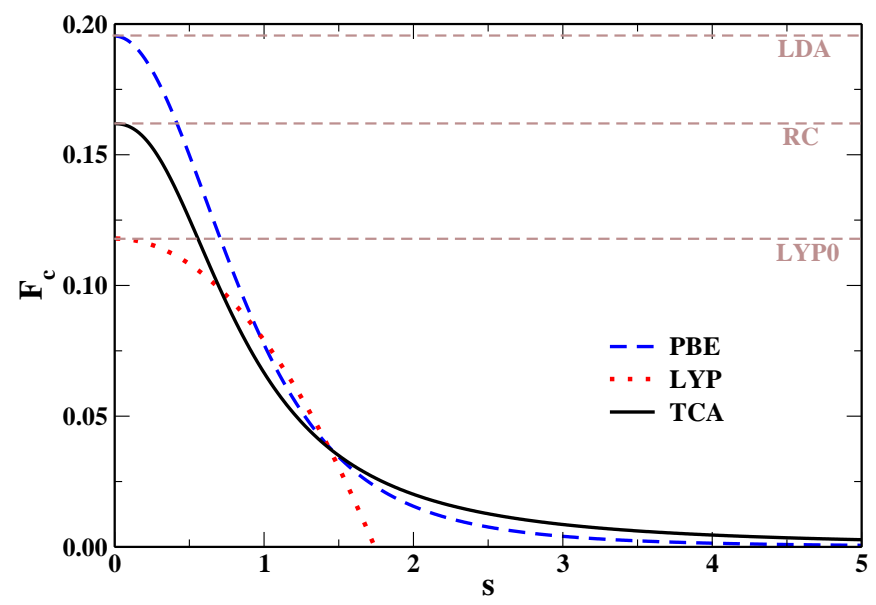

FIG. 2: Correlation enhancement factor $F_{c}$ versus the reduced gradient $s$, for $r_{s}=2$, and for $\zeta=0$ (spin-unpolarized case).

[8] recovers the exact LDA correlation, while LYP [15] recovers the local correlation present in this functional (named LYP0). We recall that both TCA and LYP are based, to some extent, on the Colle-Salvetti theory 15, 45]. Thus, a direct comparison can bring a further insight on the construction of these functionals. As shown in Fig. 2. TCA decays slower than PBE, but in a similar manner. On the other hand, the LYP correlation enhancement factor is very different especially at large gradients, where it is strongly negative. This fact implies that $\epsilon_{c}^{L Y P} \geq 0$ at large gradients, that is a formally wrong behavior. In fact, for systems dominated by large gradients, such as quasi-two-dimensional systems, the LYP total correlation can be positive, failing badly, while TCA performs as PBE.

\section{RESULTS}

\section{A. Assessment of the TCA correlation for atoms and molecules}

The TCA correlation functional has been already assessed for atomic correlation energies [17, 47]. In Ref. 17] it was shown that, for the H-Ar neutral atoms, the TCA mean absolute error (MAE) on the correlation energies is $44 \%$ and $17 \%$ smaller than in the case of $\mathrm{PBE}$ and LYP, respectively. This improvement was not confirmed for the ions $\mathrm{Li}^{+}-\mathrm{K}^{+}$: in such a case, TCA is still better than LYP, but it is slightly worse than PBE [47].

To understand better these results, we report in TableI the correlation energies for several closed- and open-shell atoms and ions as computed from different DFT functionals. We see that the local RC functional performs rather well being almost twice better than the LDA correlation, but still worse than LYP0. On the other hand, the TCA correlation functional shows an overall performance comparable to the PBEsol one and slightly worse than LYP and PBE. However, for closed-shell systems the TCA correlation performs similarly to PBEsol and better than PBE. Moreover, we highlight the fact that the TCA functional generally yields its largest errors for highly charged ions, i.e. in high-density limit cases. To some extent, this feature is shared by all the other GGA correlation functionals examined in this paper, but it appears especially pronounced for TCA and LYP.

To better understand this issue, we have performed a reduced-gradient decomposition of the TCA correlation energy [93]. This was possible as the variables in the TCA formula are factorized. We recall that a similar technique was also used to study kinetic energy functionals [94, 95]. The TCA correlation energy is thus written as

$$
E_{c}^{T C A}=\int e_{c}[n](s) B(s) d s,
$$

where the $s$-decomposed correlation energy distribution $e_{c}$ is defined by

$$
e_{c}[n](s)=\int n(\mathbf{r}) \epsilon_{c}^{R C}\left(r_{s}(\mathbf{r})\right) C(\zeta(\mathbf{r})) \delta(s(\mathbf{r})-s) d \mathbf{r} .
$$

This distribution contains all system-dependent information, whereas the enhancement factor $B$ in Eq. (9) plays the role of a universal multiplicative factor. Using Eq. (9) we can define the error function

$$
\Delta(s)=\int_{0}^{s} e_{c}\left(s^{\prime}\right) B\left(s^{\prime}\right) d s^{\prime}-E_{c}^{R e f},
$$

with $E_{c}^{R e f}$ the reference correlation energy. Thus, the error on the correlation energy is

$$
\Delta E_{c}=\Delta(\infty) .
$$

The reduced-gradient decomposition of the TCA correlation energy and the related error are reported in Fig. 
TABLE I: Correlation energy ( $\mathrm{mHa}$ ) divided by the number of electrons $\left(N_{e}\right)$ for several atoms and ions. Reference data are taken from Refs. 91] and 92]. The last lines report the mean error (ME), the mean absolute error (MAE), and the mean absolute relative error (MARE).

\begin{tabular}{|c|c|c|c|c|c|c|c|c|c|c|}
\hline \multirow[b]{2}{*}{ Atom } & \multirow[b]{2}{*}{$N_{e}$} & \multicolumn{3}{|c|}{ local functionals } & \multicolumn{5}{|c|}{ semilocal functionals } & \multirow[b]{2}{*}{ Ref. } \\
\hline & & $\mathrm{LDA}$ & LYP0 & $\overline{\mathrm{RC}}$ & $\mathrm{PBE}$ & PBEint & PBEsol & LYP & $\overline{\mathrm{TCA}}$ & \\
\hline $\mathrm{He}$ & 2 & -56.2 & -36.1 & -47.4 & -21.0 & -24.5 & -26.3 & -21.9 & -22.4 & -21.0 \\
\hline $\mathrm{Li}^{+}$ & 2 & -67.3 & -43.7 & -56.2 & -22.4 & -26.3 & -28.3 & -23.8 & -26.4 & -21.7 \\
\hline $\mathrm{Be}^{2+}$ & 2 & -75.2 & -48.2 & -61.4 & -23.0 & -27.2 & -29.3 & -24.5 & -28.6 & -22.2 \\
\hline $\mathrm{Be}$ & 4 & -56.0 & -34.3 & -45.1 & -21.4 & -24.6 & -26.1 & -23.6 & -22.2 & -23.6 \\
\hline $\mathrm{B}^{+}$ & 4 & -63.0 & -39.0 & -50.7 & -23.0 & -26.5 & -28.2 & -26.7 & -25.1 & -27.8 \\
\hline $\mathrm{C}^{2+}$ & 4 & -68.5 & -42.4 & -54.7 & -24.0 & -27.7 & -29.5 & -28.6 & -27.2 & -35.1 \\
\hline $\mathrm{N}^{3+}$ & 4 & -73.0 & -45.1 & -57.8 & -24.7 & -28.6 & -30.5 & -30.0 & -28.8 & -35.1 \\
\hline $\mathrm{O}^{4+}$ & 4 & -76.9 & -47.3 & -60.3 & -25.3 & -29.2 & -31.2 & -30.9 & -30.0 & -38.5 \\
\hline $\mathrm{Ar}^{8+}$ & 10 & -96.8 & -57.1 & -71.4 & -41.0 & -46.1 & -48.5 & -44.9 & -46.6 & -39.9 \\
\hline $\mathrm{Ne}$ & 10 & -74.3 & -46.7 & -59.7 & -35.1 & -39.2 & -41.2 & -38.4 & -37.9 & -39.1 \\
\hline $\mathrm{Ar}^{6+}$ & 12 & -90.2 & -53.5 & -67.4 & -38.3 & -43.2 & -45.6 & -44.8 & -43.1 & -41.3 \\
\hline $\mathrm{Ar}$ & 18 & -79.1 & -47.9 & -61.0 & -39.3 & -43.5 & -45.5 & -41.7 & -41.5 & -40.1 \\
\hline $\mathrm{Kr}$ & 36 & -90.8 & -52.8 & -66.6 & -49.1 & -53.8 & -56.0 & -48.6 & -50.4 & -57.4 \\
\hline $\mathrm{Zn}$ & 30 & -88.5 & -52.3 & -66.0 & -46.9 & -51.5 & -53.7 & -47.7 & -48.7 & -56.2 \\
\hline $\mathrm{ME}$ & & -39.8 & -10.5 & -23.3 & 4.6 & 0.5 & -1.5 & 1.6 & 1.4 & \\
\hline MAE & & 39.8 & 11.7 & 23.3 & 5.0 & 4.2 & 4.6 & 3.8 & 4.6 & \\
\hline MARE & & $124.81 \%$ & $41.05 \%$ & $78.03 \%$ & $13.02 \%$ & $12.64 \%$ & $14.69 \%$ & $9.92 \%$ & $13.47 \%$ & \\
\hline \multicolumn{11}{|c|}{ Open-shell atoms } \\
\hline $\mathrm{Ne}^{7+}$ & 3 & -80.4 & -41.8 & -59.4 & -19.4 & -23.2 & -25.2 & -26.9 & -27.1 & -17.0 \\
\hline $\mathrm{Be}^{+}$ & 3 & -57.6 & -33.3 & -46.6 & -18.1 & -21.3 & -23.0 & -20.4 & -21.7 & -15.8 \\
\hline $\mathrm{Li}$ & 3 & -50.3 & -29.7 & -41.2 & -17.1 & -20.1 & -21.6 & -17.8 & -19.3 & -15.1 \\
\hline $\mathrm{Ar}^{15+}$ & 3 & -94.9 & -44.9 & -64.3 & -19.7 & -23.7 & -25.8 & -29.2 & -29.1 & -17.4 \\
\hline $\mathrm{C}^{3+}$ & 3 & -67.7 & -37.7 & -53.1 & -18.9 & -22.5 & -24.3 & -23.7 & -24.5 & -16.5 \\
\hline $\mathrm{N}^{4+}$ & 3 & -71.5 & -39.1 & -55.2 & -19.1 & -22.8 & -24.7 & -24.8 & -25.4 & -16.7 \\
\hline $\mathrm{B}^{2+}$ & 3 & -63.2 & -35.8 & -50.3 & -18.6 & -22.0 & -23.8 & -22.3 & -23.3 & -16.2 \\
\hline $\mathrm{O}^{5+}$ & 3 & -74.9 & -40.1 & -56.9 & -19.2 & -23.0 & -24.9 & -25.6 & -26.0 & -16.8 \\
\hline $\mathrm{O}^{+}$ & 7 & -65.6 & -38.1 & -52.8 & -27.0 & -30.6 & -32.4 & -29.5 & -30.5 & -27.7 \\
\hline $\mathrm{N}$ & 7 & -61.0 & -35.3 & -49.4 & -25.7 & -29.1 & -30.8 & -27.4 & -28.2 & -26.9 \\
\hline $\mathrm{ME}$ & & -50.1 & -19.0 & -34.3 & -1.6 & -5.2 & -7.0 & -6.1 & -6.9 & \\
\hline MAE & & 50.1 & 19.0 & 34.3 & 2.0 & 5.2 & 7.0 & 6.1 & 6.9 & \\
\hline MARE & & $285.35 \%$ & $110.24 \%$ & $196.27 \%$ & $11.92 \%$ & $30.48 \%$ & $40.69 \%$ & $36.31 \%$ & $40.53 \%$ & \\
\hline \multicolumn{11}{|c|}{ Overall statistics } \\
\hline $\mathrm{ME}$ & & -44.1 & -14.0 & -27.9 & 2.0 & -1.9 & -3.8 & -1.6 & -2.0 & \\
\hline MAE & & 44.1 & 14.8 & 27.9 & 3.8 & 4.6 & 5.6 & 4.8 & 5.6 & \\
\hline MARE & & $191.70 \%$ & $69.87 \%$ & $127.30 \%$ & $12.56 \%$ & $20.07 \%$ & $25.52 \%$ & $20.91 \%$ & $24.75 \%$ & \\
\hline
\end{tabular}

3 for the relevant cases of the $\mathrm{Be}$ atom and the $\mathrm{O}^{4+}$ ion. Both systems have 4 electrons but in the former case TCA performs quite well, while it gives a relatively large error for $\mathrm{O}^{4+}$. We see that both systems display a similar overall shape for the $s$-decomposed correlation energy distribution, but the the curve is more structured for $s>1$ for the $\mathrm{O}^{4+}$ ion (top panel). However, the enhance- ment factor $B$ decays in a rather fast way $(B(1) \approx 0.4$ and $B(2) \approx 0.2)$ thus most features at $s>1$ are largely dumped and the two curves for $e_{c} B$ are very similar (middle panel). As a consequence the error functions $\Delta(s)$ display similar shapes and slopes for both $\mathrm{Be}$ and $\mathrm{O}^{4+}$ (bottom panel). The only notable difference between the two curves is therefore the reference correlation energy, 


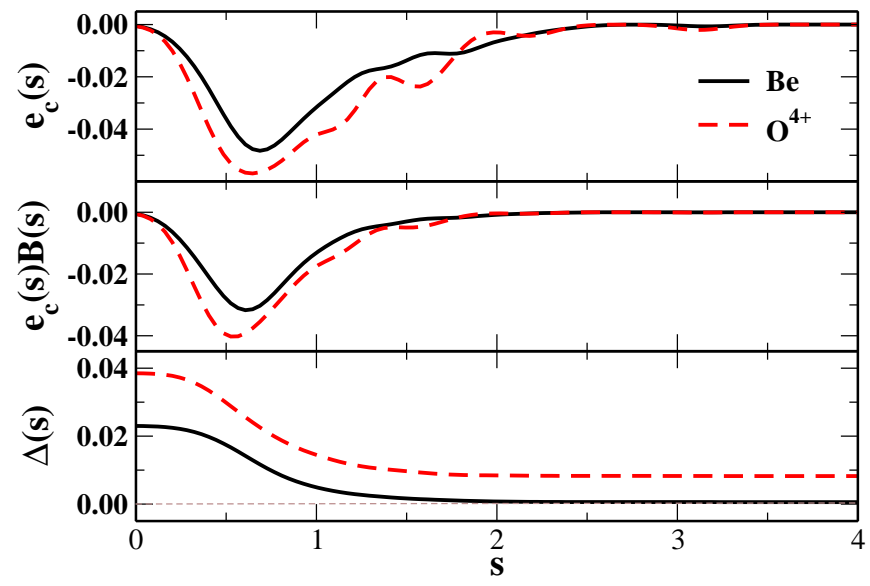

FIG. 3: Reduced-gradient decomposition of the TCA correlation energy and its error for the Be atom and the $\mathrm{O}^{4+}$ ion.

which is larger for $\mathrm{O}^{4+}$ than for $\mathrm{Be}$, causing a shift of the two lines. As a consequence for $s \rightarrow \infty$ the Be curve tends to zero, while the $\mathrm{O}^{4+}$ does not, differing from it approximately by the difference between the $\mathrm{O}^{4+}$ and $\mathrm{Be}$ reference correlation energies.

This analysis suggests that the limitations of the TCA correlation functional for highly-charged ions depend mainly on a too fast decay of the enhancement factor, whereas the RC local functional plays a minor role. Anyway, we remark that Be isoelectronic series is a difficult example of strong correlation in the high-density limit [60], and all semilocal correlation functionals perform poorly in this case.

As an additional test for the TCA correlation functional we report in Table II the correlation energies for several closed-shell molecules as computed with different semilocal functionals (local functionals are not reported here because they perform poorly). In this case we find that TCA performs very well yielding the smallest MAE and MARE, although these are very close to the PBE and LYP ones.

The results of this section indicate that, for atomic and molecular systems, the TCA correlation functional is competitive with the popular PBE and LYP ones. This is not highly surprising, considering that the TCA functional have been constructed using atomic systems as references. Nevertheless, the present assessment provides a more quantitative indication on this behavior, confirming that the use of TCA for computational chemistry problems is a viable option.

\section{B. Assessment of TCA correlation for jellium model systems}

Having assessed the TCA correlation functional for atoms and molecules we consider here the opposite limit and test the performance of the functional for the jellium model. Thus, we report in Table III the aver-
TABLE II: Correlation energy $(\mathrm{mHa})$ for several closed-shell molecules. Reference data are taken from Ref. [96]. The last lines report the mean error (ME), the mean absolute error (MAE), and the mean absolute relative error (MARE).

\begin{tabular}{lrrrrrr}
\hline \hline Molecule & PBE & PBEint & PBEsol & LYP & TCA & Ref \\
\hline $\mathrm{H}_{2}$ & -44 & -49 & -52 & -38 & -41 & -41 \\
$\mathrm{LiH}$ & -85 & -98 & -105 & -89 & -88 & -83 \\
$\mathrm{Li}_{2}$ & -125 & -144 & -153 & -133 & -130 & -124 \\
$\mathrm{CH}_{2}$ & -225 & -251 & -264 & -232 & -228 & -239 \\
$\mathrm{CH}_{4}$ & -301 & -332 & -348 & -294 & -298 & -299 \\
$\mathrm{H}_{2} \mathrm{O}$ & -329 & -364 & -382 & -341 & -339 & -371 \\
$\mathrm{FH}$ & -339 & -378 & -396 & -363 & -359 & -389 \\
$\mathrm{HCN}$ & -442 & -490 & -514 & -463 & -455 & -515 \\
$\mathrm{CO}$ & -451 & -502 & -527 & -484 & -472 & -535 \\
$\mathrm{CO}_{2}$ & -744 & -824 & -863 & -790 & -777 & -535 \\
$\mathrm{~N}_{2}$ & -454 & -504 & -528 & -483 & -472 & -549 \\
$\mathrm{SiH}_{2}$ & -555 & -619 & -649 & -597 & -583 & -567 \\
$\mathrm{SiH}_{4}$ & -615 & -685 & -718 & -648 & -639 & -606 \\
$\mathrm{PH}_{3}$ & -643 & -713 & -747 & -675 & -668 & -652 \\
$\mathrm{ClH}$ & -688 & -762 & -797 & -727 & -723 & -707 \\
& & & & & & \\
$\mathrm{ME}$ & 12 & -34 & -55 & -10 & -4 & \\
$\mathrm{MAE}$ & 42 & 50 & 59 & 42 & 41 & \\
$\mathrm{MARE}$ & $8.9 \%$ & $12.5 \%$ & $15.7 \%$ & $9.2 \%$ & $8.6 \%$ & \\
\hline \hline
\end{tabular}

age relative errors of the correlation energy with respect to diffusion Monte Carlo (DMC) benchmark values 54 $\left(\left(E_{c}-E_{c}^{D M C}\right) / E_{c}^{D M C}\right)$ for magic jellium spheres with $N=2,8,18,20,34,40,58,92$ and 106 and several bulk parameter values (our results agree within $1 \mathrm{mHa}$ with the ones of Ref. [54]).

We see that the $\mathrm{RC}$ results are remarkably accurate, being smaller than the exact ones for $r_{s}=1$ and $r_{s}=2$ and larger than the exact ones for $r_{s} \geq 3$. The signed average error is $1.2 \%$. Much larger errors are found for the other local functionals, including LDA, which is exact for bulk jellium but rather poor for other density regimes. Concerning the GGA functionals, we note that all the correlation functionals that recover the exact LDA (PBE, PBEint, PBEsol) give correlation energies smaller than the DMC results, whereas TCA and LYP results are all larger than the DMC ones. In the case of TCA this behavior is due to the fact that $\mathrm{RC}$ results are already very accurate and $E_{c}^{T C A} \geq E_{c}^{R C}$. In analogy with the case of highly charged ions, this indicates a too fast decay of the TCA enhancement factor in rapidly-varying regions. Consequently, TCA results are not very good for jellium clusters, although they are also not far from PBEsol ones.

Total correlation energies, however, are not the only important feature to consider in jellium clusters. Instead, energy differences are often more important quantities to consider. In particular, we can mention the description of 
TABLE III: Average relative errors of the correlation energy $\left(\left(E_{c}-E_{c}^{D M C}\right) / E_{c}^{D M C}\right)$ of magic jellium spheres (with $N=2,8$, 18, 20, 34, 40, 58, 92 and 106), for several bulk parameters. The fixed-node corrected DMC data are taken from Ref. [54].

\begin{tabular}{|c|c|c|c|c|c|c|c|c|}
\hline \multirow[b]{2}{*}{$r_{s}$} & \multicolumn{3}{|c|}{ local functionals } & \multicolumn{5}{|c|}{ semilocal functionals } \\
\hline & $\mathrm{LDA}$ & $\mathrm{RC}$ & LYP0 & $\mathrm{PBE}$ & PBEsol & PBEint & TCA & LYP \\
\hline 1 & 40.5 & 18.5 & -11.3 & 6.8 & 13.3 & 11.3 & -6.5 & -22.0 \\
\hline 2 & 34.2 & 8.1 & -22.5 & 7.9 & 13.0 & 11.4 & -12.5 & -29.6 \\
\hline 3.25 & 29.9 & -1.7 & -32.2 & 7.4 & 11.9 & 10.5 & -19.5 & -36.4 \\
\hline 4 & 28.1 & -6.3 & -36.3 & 7.1 & 11.2 & 9.9 & -22.9 & -39.5 \\
\hline 5.26 & 26.8 & -12.6 & -41.9 & 7.8 & 11.5 & 10.3 & -27.6 & -43.6 \\
\hline Average & 31.9 & 1.2 & -28.8 & 7.4 & 12.2 & 10.7 & -17.8 & -34.2 \\
\hline
\end{tabular}

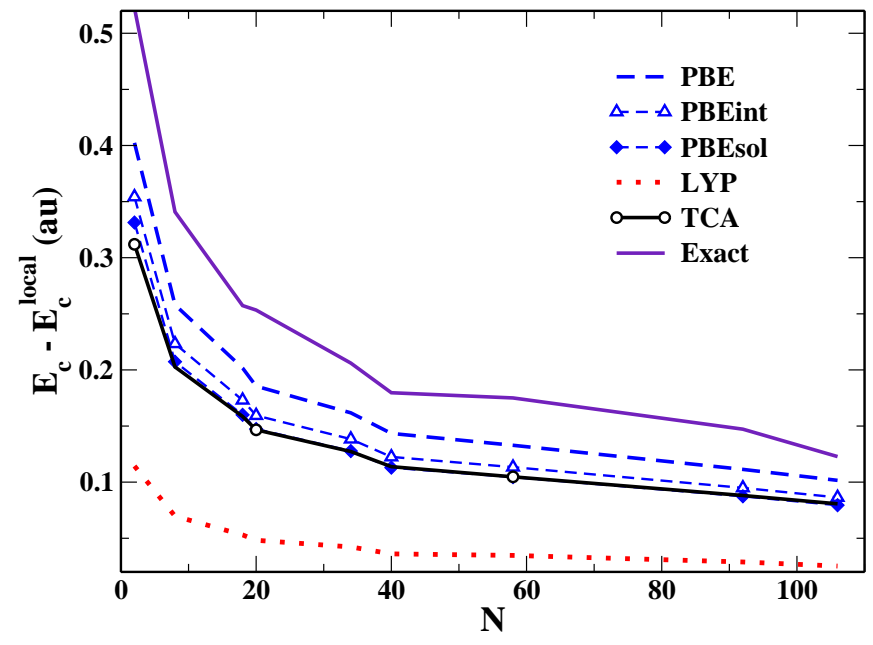

FIG. 4: $\quad E_{c}-E_{c}^{\text {local }}$ for magic jellium spheres with $r_{s}=2$, and $N=2,8,18,20,34,40,58,92$ and 106 .

the non-local effects, which are dominated by quantum oscillations and surface effects and play a fundamental role in many cases. To investigate this aspect we report in Fig. 4 the quantity $E_{c}-E_{c}^{\text {local }}$ for magic jellium spheres with $r_{s}=2$, and $N=2,8,18,20,34,40,58$, 92 and 106. We see that, in this case, all GGA functionals, except LYP, perform quite similarly. The TCA results are almost the same as the PBEsol ones (the two lines are superimposed in the plot) and are very close to those given by PBEint. Slightly better results are given by PBE.

To complete our assessment based on the jellium model, we report in Table IV semi-infinite jellium surfaces correlation energies as computed with several functionals. Inspection of the data shows that all the local functionals are rather inaccurate for this problem. Nevertheless, it is interesting to remark that, even if both RC and LYP0 are based on the Colle-Salvetti theory, the RC functional gives results very similar to LDA, while LYP0 is even more inaccurate.

Definitely improved results are given by all the GGA functionals. Among these, the TCA correlation func- tional yields the best performance, slightly outperforming even PBEsol that was fitted to this property. On the other hand, PBE gives larger results, while LYP strongly underestimates these jellium surface correlation energies.

The results of this section indicate that the TCA correlation is not very accurate for total energies of jellium models, probably because it does not recover the exact LDA limit. Nevertheless, it performs surprisingly well for energy differences, such as in the case of semi-infinite jellium surface correlation energies, being at least competitive with more popular functionals for solid-state such as PBEsol. These results suggest that the TCA correlation, in contrast to the LYP one, can also be used in calculations where the slowly-varying density regime is relevant.

\section{Exchange-correlation functionals}

The results of the previous subsections indicate that the TCA correlation functional can be a valid tool both for computational chemistry and solid-state calculations. However, to obtain a practical tool for such applications it is necessary to couple the TCA correlation with an appropriate exchange functional. Previous studies [17, 48 51] showed that TCA works well with PBE exchange for molecular problems but not for solid-state; on the opposite, it performs well in solid-state together with PBEsol exchange but, in this case, it does not yield very good results for chemical tests. Some sort of compromise seems therefore necessary to ensure good accuracy for all problems and obtain an XC functional of broad applicability. To investigate this issue we consider here the performance of the TCA correlation functional associated with a family of exchange functionals whose enhancement factor (defined as $F_{x}=\epsilon_{x} / \epsilon_{x}^{L D A}$ with $\epsilon_{x}$ the exchange energy per particle) has the general form

$$
F_{x}(s, \alpha)=1+\kappa-\frac{\kappa}{1+\frac{\mu(s, \alpha)}{\kappa} s^{2}}
$$

where

$$
\mu(s, \alpha)=\mu^{G E 2}+\left(\mu^{P B E}-\mu^{G E 2}\right) \frac{\alpha s^{2}}{1+\alpha s^{2}},
$$


TABLE IV: Semi-infinite jellium surface correlation energies $\left(\mathrm{erg} / \mathrm{cm}^{2}\right)$ for different values of the bulk parameter $r_{s}$ as computed with different functionals. Diffusion Monte Carlo (DMC) results 23] are also given as reference. The results within the DMC error uncertainty are highlighted in bold style.

\begin{tabular}{|c|c|c|c|c|c|c|c|c|c|}
\hline \multirow[b]{2}{*}{$r_{s}$} & \multicolumn{3}{|c|}{ local functionals } & \multicolumn{5}{|c|}{ semilocal functionals } & \multirow[b]{2}{*}{$\mathrm{DMC}$} \\
\hline & LDA & $\mathrm{RC}$ & LYP0 & PBE & PBEint & PBEsol & TCA & $\overline{L Y P}$ & \\
\hline$\overline{2}$ & 318 & 325 & 267 & 827 & 745 & 708 & 734 & 388 & $768 \pm 50$ \\
\hline 3 & 95 & 96 & 74 & 275 & 234 & 246 & 243 & 109 & $242 \pm 10$ \\
\hline 4 & 39 & 38 & 27 & 124 & 111 & 105 & 108 & 40 & $104 \pm 8$ \\
\hline 6 & 10 & 9 & 6 & 40 & 35 & 33 & 33 & 8 & $33 \pm \cdots$ \\
\hline
\end{tabular}

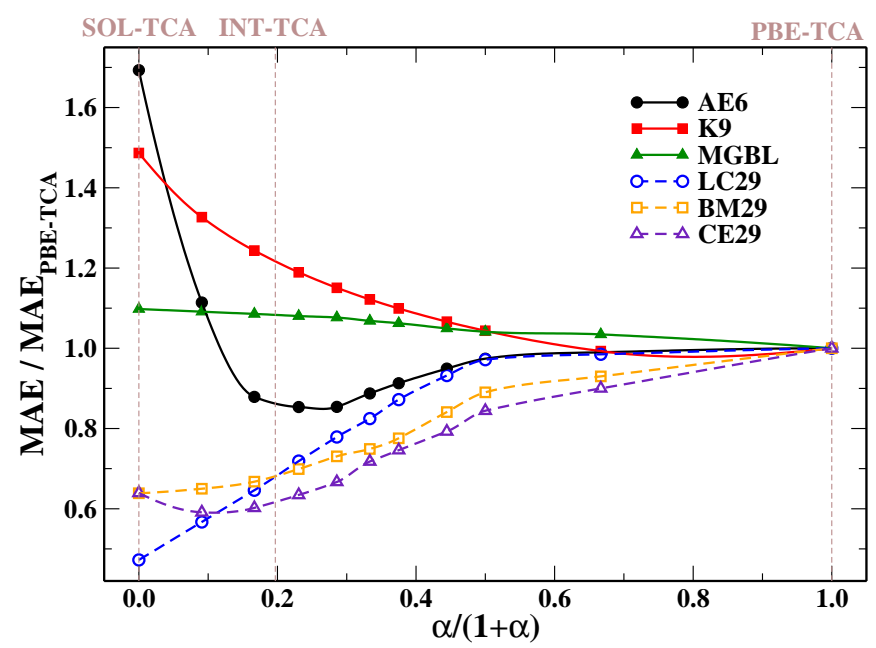

FIG. 5: Mean absolute errors (MAE) relative to the PBETCA one, computed with the XC functional using the exchange of Eq. (13) with different values of $\alpha$ and the TCA correlation, for several properties: atomization energies of small molecules (AE6) 61, 62], barrier heights and reaction energies (K9) [62, 70], bond lengths of small molecules (MGBL) [71], lattice constants (LC29), bulk moduli (BM29), and cohesive energies (CE29) of 29 solids [79, 80].

with $\mu^{G E 2}=10 / 81, \mu^{P B E}=0.21951$, and $\alpha$ being a parameter going from 0 to $+\infty$ which controls the bias of the functional towards the slowly- or the rapidly-varying density regime. This family includes as limiting cases the PBEsol exchange $(\alpha=0)$ and the PBE exchange $(\alpha=+\infty)$. For $\alpha=0.197$ it recovers instead the PBEint exchange functional [11].

In Fig. 5 we report the mean absolute errors divided by the PBE-TCA one, as functions of $\alpha$, for the family of $\mathrm{XC}$ functionals build from the exchange of Eq. (13) and the TCA correlation. Several properties are considered: atomization energies of small molecules (AE6) 61, 62], barrier heights and reaction energies (K9) 62, 70], bond lengths of small molecules (MGBL) [71], lattice constants (LC29), bulk moduli (BM29), and cohesive energies (CE29) of 29 solids [79, 80]. The plot confirms the findings of previous investigations about PBE-TCA and SOL-TCA. In addition, it shows that the evolution with $\alpha$ is not monotonic for all properties. In fact, for atomization and cohesive energies minima located approximately at $\alpha=0.11$ and $\alpha=0.25$ are observed. This fact, together with the opposite behavior of molecular and solid-state properties, indicates that a "best" compromise may only be found for some intermediate values of $\alpha$. We observe that the INT-TCA functional seems to be close to such "best" choice. Thus, we simply assume the INT-TCA functional as our guess for the "best compromise".

The results of Fig. 5 are also confirmed when additional systems and properties are analyzed. This is the case of Table $\nabla$ where we report the MAEs of different tests of relevance for computational chemistry and solid-state physics as obtained using the PBE, PBEint and PBEsol functionals as well as their TCA variants. The data show that indeed PBE-TCA is the best functional for properties related to computational chemistry (LRAE of -12.7), being especially good for thermochemistry, structural properties, and non-covalent interactions. On the contrary, PBE-TCA is the worst functional for solid-state properties. An opposite behavior is found for SOL-TCA, which displays the best performance for solid-state tests (LRAE=-9.5), outperforming also the original PBEsol; however, it shows rather poor results for chemistry-related problems (LRAE=0.5). Note that anyway SOL-TCA performs in this case definitely better than PBEsol and even better than PBEint (see below). Finally, INT-TCA shows a performance close to PBETCA for computational chemistry and close to SOL-TCA for solid-state, being on average the best functional.

A further interesting feature to observe is the comparison between the original functionals using PBE-like correlation and their corresponding variant using the TCA correlation. In this way, it is actually possible to understand whether the use of TCA may bring some advantages for a given choice of exchange and a given property. Such a comparison is summarized in Table $\nabla$ by the quantity denoted $\Delta$ which corresponds, for each line, to the difference between the -TCA value and the PBE-like one. Thus, negative values of $\Delta$ indicate that the use of TCA improves the results, while positive values denote the opposite. The values of $\Delta$ in the table show that the use of TCA correlation systematically improves the description 
TABLE V: Mean absolute errors for different tests, as obtained from various exchange-correlation functionals. For each couple of functionals the difference between the value obtained using the TCA and the original PBE-like correlation is reported in the columns denoted by $\Delta$. The logarithmic relative absolute error (LRAE; Eq. (10) is also reported at the bottom of each group of tests as well as for the global set.

\begin{tabular}{|c|c|c|c|c|c|c|c|c|c|}
\hline Test & $\mathrm{PBE}$ & PBE-TCA & $(\Delta)$ & PBEint & INT-TCA & $(\Delta)$ & PBEsol & SOL-TCA & $(\Delta)$ \\
\hline \multicolumn{10}{|c|}{ Main group thermochemistry $(\mathrm{kcal} / \mathrm{mol})$} \\
\hline Atomiz. energy (AE6) & 13.35 & 10.59 & $(-2.76)$ & 23.60 & 9.37 & $(-14.22)$ & 33.21 & 17.95 & $(-15.26)$ \\
\hline Atomiz. energy (W4) & 10.72 & 7.82 & $(-2.90)$ & 15.49 & 9.37 & $(-6.12)$ & 21.35 & 12.68 & $(-8.67)$ \\
\hline Atomiz. energy (G2/97) & 14.76 & 9.89 & $(-4.87)$ & 26.44 & 11.14 & $(-15.30)$ & 37.69 & 19.05 & $(-18.63)$ \\
\hline Reaction energy (BH76RC) & 4.35 & 3.96 & $(-0.40)$ & 5.38 & 5.14 & $(-0.24)$ & 6.31 & 6.21 & $(-0.10)$ \\
\hline Reaction energy (OMRE) & 6.74 & 7.05 & $(0.32)$ & 8.05 & 6.32 & $(-1.73)$ & 11.85 & 9.53 & $(-2.32)$ \\
\hline Barrier heights (BH76) & 9.77 & 8.72 & $(-1.05)$ & 10.90 & 9.55 & $(-1.35)$ & 12.17 & 10.71 & $(-1.45)$ \\
\hline Kinetics (K9) & 7.47 & 6.45 & $(-1.01)$ & 9.05 & 8.04 & $(-1.01)$ & 10.55 & 9.59 & $(-0.96)$ \\
\hline LRAE & -4.1 & -11.9 & $(-7.8)$ & 9.6 & -8.0 & $(-17.5)$ & 20.9 & 6.8 & $(-14.1)$ \\
\hline \multicolumn{10}{|c|}{ Main group geometry $\left(\mathrm{m} \AA\right.$ and $\left.\mathrm{cm}^{-1}\right)$} \\
\hline H bond lengths (MGHBL9) & 11.45 & 4.77 & $(-6.67)$ & 14.72 & 6.95 & $(-7.77)$ & 14.53 & 6.99 & $(-7.54)$ \\
\hline non-H bond lengths (MGNHBL11) & 7.64 & 7.44 & $(-0.20)$ & 7.18 & 6.89 & $(-0.29)$ & 5.19 & 6.04 & $(0.85)$ \\
\hline vibrations (F38) & 58.38 & 36.63 & $(-21.75)$ & 66.65 & 40.27 & $(-26.38)$ & 67.54 & 39.54 & $(-28.00)$ \\
\hline LRAE & 8.2 & -11.6 & $(-19.8)$ & 12.9 & -5.9 & $(-18.8)$ & 8.2 & -8.0 & $(-16.2)$ \\
\hline \multicolumn{10}{|c|}{ Transition metals (kcal/mol, mÅ, and $\mathrm{kcal} /(\mathrm{mol} \cdot$ atom $)$ for AUnAE) } \\
\hline Atomiz. energy (TM10AE) & 13.02 & 11.04 & $(-1.98)$ & 15.20 & 11.16 & $(-4.04)$ & 18.34 & 12.66 & $(-5.68)$ \\
\hline Reaction energy (TMRE) & 3.73 & 3.20 & $(-0.53)$ & 6.94 & 4.64 & $(-2.29)$ & 9.89 & 7.59 & $(-2.30)$ \\
\hline Gold cluster AE (AUnAE) & 0.60 & 2.30 & $(1.70)$ & 1.50 & 0.81 & $(-0.69)$ & 3.63 & 1.61 & $(-2.02)$ \\
\hline Bond lengths (TMBL) & 13.51 & 10.43 & $(-3.08)$ & 17.28 & 17.01 & $(-0.28)$ & 22.42 & 24.23 & $(1.81)$ \\
\hline Gold clusters BL (AuBL6) & 56.46 & 31.42 & $(-25.03)$ & 22.12 & 25.63 & $(3.51)$ & 21.11 & 25.74 & $(4.63)$ \\
\hline LRAE & -8.8 & -7.2 & $(1.6)$ & -0.1 & -10.4 & $(-10.3)$ & 14.1 & 4.0 & $(-10.1)$ \\
\hline \multicolumn{10}{|c|}{ Non-covalent interactions $(\mathrm{kcal} / \mathrm{mol})$} \\
\hline Hydrogen bonds (HB6) & 0.38 & 0.46 & $(0.08)$ & 0.50 & 0.62 & $(0.12)$ & 1.65 & 0.67 & $(-0.98)$ \\
\hline Dipole-dipole (DI6) & 0.38 & 0.46 & $(0.08)$ & 0.44 & 0.57 & $(0.13)$ & 0.96 & 0.47 & $(-0.49)$ \\
\hline Dihydrogen bonds (DHB23) & 0.98 & 0.68 & $(-0.29)$ & 1.01 & 0.66 & $(-0.35)$ & 1.76 & 1.08 & $(-0.68)$ \\
\hline LRAE & -23.0 & -22.7 & $(0.3)$ & -16.4 & -15.7 & $(0.7)$ & 20.2 & -10.1 & $(-30.4)$ \\
\hline \multicolumn{10}{|c|}{ Other molecular properties $(\mathrm{kcal} / \mathrm{mol})$} \\
\hline Multireference AE (W4-MR) & 21.80 & 14.90 & $(-6.90)$ & 28.98 & 21.68 & $(-7.29)$ & 35.50 & 27.74 & $(-7.77)$ \\
\hline Difficult cases (DC9/12) & 40.75 & 29.78 & $(-10.97)$ & 63.48 & 35.00 & $(-28.47)$ & 82.94 & 53.18 & $(-29.76)$ \\
\hline Small interfaces (SI12) & 3.72 & 6.45 & $(2.72)$ & 2.69 & 3.38 & $(0.69)$ & 3.79 & 2.76 & $(-1.03)$ \\
\hline LRAE & -4.1 & -6.2 & $(-2.1)$ & 1.7 & -7.8 & $(-9.6)$ & 13.5 & -1.1 & $(-14.6)$ \\
\hline \multicolumn{10}{|c|}{ Solid-state (mA, GPa, and eV) } \\
\hline Lattice constants (LC29) & 53.00 & 63.29 & $(10.28)$ & 36.05 & 40.72 & $(4.67)$ & 30.99 & 29.84 & $(-1.14)$ \\
\hline Bulk moduli (BM29) & 12.43 & 13.87 & $(1.43)$ & 8.87 & 9.26 & $(0.39)$ & 8.14 & 8.86 & $(0.71)$ \\
\hline Cohesive energies (CE29) & 0.13 & 0.32 & $(0.19)$ & 0.20 & 0.20 & $(0.00)$ & 0.34 & 0.21 & $(-0.13)$ \\
\hline LRAE & -3.0 & 13.9 & $(16.9)$ & -8.1 & -5.6 & $(2.5)$ & -3.5 & -9.9 & $(-6.4)$ \\
\hline \multicolumn{10}{|c|}{ Overall statistics } \\
\hline Chemistry LRAE & -6.8 & -12.7 & $(-5.9)$ & 3.2 & -10.3 & $(-13.5)$ & 18.0 & 0.5 & $(-17.5)$ \\
\hline Solid-state LRAE & -2.7 & 14.2 & $(16.9)$ & -7.8 & -5.3 & $(2.5)$ & -3.2 & -9.5 & $(-6.4)$ \\
\hline Average LRAE & -4.9 & 0.6 & $(5.5)$ & -2.5 & -8.0 & $(-5.5)$ & 7.3 & -4.7 & $(-12.0)$ \\
\hline
\end{tabular}


of chemistry-related properties, with only few exceptions. This behavior is the same for all the considered exchange functionals. On the contrary, in general the description of solid-state properties is slightly worsened when TCA correlation is used. This is not true, however, for the PBEsol exchange, since in this case both lattice constants and bulk moduli are left basically unchanged (within the numerical precision) while the cohesive energies are improved. Summarizing, we can say that, when TCA correlation is used in association with PBEint or PBEsol exchange, the improvement for computational chemistry tests is bigger than the worsening for solid-state tests (if any). Hence, these TCA-based functionals perform better than their original counterparts. Oppositely, this is not the case when PBE exchange is used. In such a case, the PBE-TCA large improvement in chemistry-related properties is accompanied by a significant worsening in solid-state tests.

To complete our investigation on the compatibility of the TCA correlation with GGA exchange functionals, we consider its use with functionals not belonging to the family defined in Eq. (13). Of course, there exists a huge number of GGA exchange functionals and any choice will be invariably arbitrary. Nevertheless, to take an easy option, we have selected a few functionals that have originally been developed as exchange-only functionals, namely B88 [14], OPTX [59], Wu-Cohen [20], and $\mathrm{PBEsol}_{b}[60]$. These have been used to form the corresponding XC functionals B-TCA, O-TCA, WC-TCA, and $\mathrm{SOL}_{b}$-TCA. The performance of these functionals for our suite of tests is reported in Table VI. Inspection of the table shows that both B-TCA and O-TCA perform in general rather poorly with the notable exception of structural properties where they yield the best results among all the functionals considered in this paper. On the other hand, WC-TCA displays a very good performance for most tests ( $\mathrm{LRAE}=-8)$. This value is similar to the one of INT-TCA. This similarity may be rationalized considering that both the exchange functionals perform an interpolation between the slowly- and the rapidly-varying density regime. Finally, we note that $\mathrm{SOL}_{b}$-TCA gives a global LRAE worse than INT-TCA or WC-TCA but rather close to SOL-TCA. Nevertheless, unlike the latter, $\mathrm{SOL}_{b}$-TCA shows a very balanced performance among all tests, with no evident failures. Thus, INT-TCA, WC-TCA, and $\mathrm{SOL}_{b}$-TCA are the only XC functionals displaying a negative LRAE both for chemistry and solid-state tests.

\section{EXAMPLE APPLICATIONS}

To conclude our work, we discuss two applications where TCA correlation can be fruitfully employed. To do this, we apply the INT-TCA, WC-TCA, and SOL $b$-TCA functionals (the ones displaying negative LRAE values for both chemistry and solid-state) to two challenging tests for GGA functionals: the calculation of chemisorp-
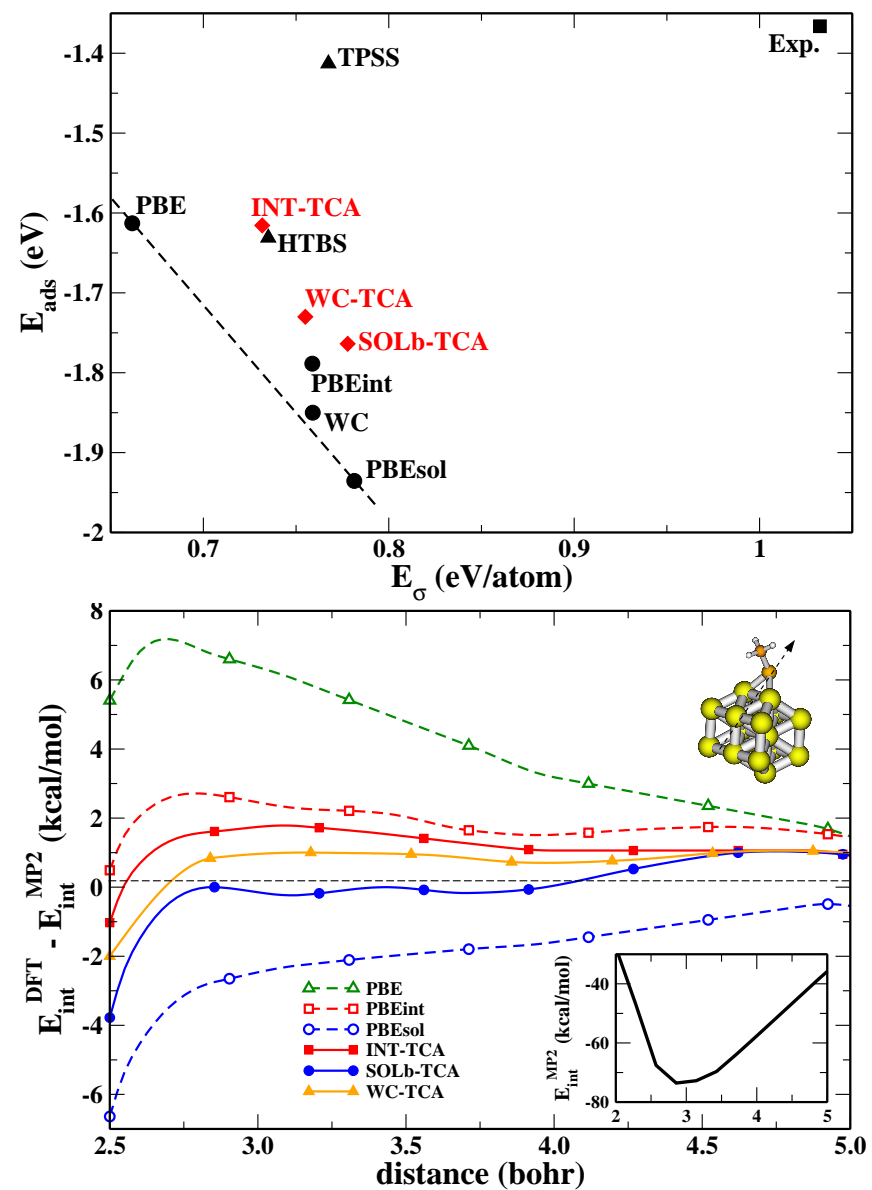

FIG. 6: Upper panel: chemisorption energy of CO on $\operatorname{Pt}(111)$ as a function of the corresponding surface energy as obtained from several functionals. The dashed line indicates the usual behavior of most GGA functionals. Data different from INT-TCA, WC-TCA, and $\mathrm{SOL}_{b}$-TCA ones are taken from Refs. [87, 97]. Lower panel: interaction energy errors for a methylthiolate molecule on a $\mathrm{Cu}_{17}$ cluster as resulting from different functionals. Reference data are MP2 results [1].

tion energies $E_{a d s}$ of $\mathrm{CO}$ on $\mathrm{Pt}(111)$ surfaces vs the corresponding surface energies $E_{\sigma}$ [87, 97] and the computation of the interaction energy between a methylthiolate molecule and a copper cluster $\mathrm{Cu}_{17}$ [11]. These problems require good accuracy for both chemical and solidstate properties and are usually poorly described by GGA functionals, which turn out to be too "specialized" to yield the required balanced description of the problem.

The results of our calculations are shown in Fig. 6. The upper panel reports the chemisorption energy of CO on $\mathrm{Pt}(111)$ versus the corresponding surface energy as obtained from several functionals. As shown in previous works [87, 97], the results of most GGA functionals tend to fall on a straight line, roughly defined by the PBE and PBEsol points (dashed line in the figure). This happens because, in most cases, at the GGA level, improvements for the adsorption energy come at the expense of 
TABLE VI: Mean absolute errors for different tests, as obtained from various exchange-correlation functionals using the TCA correlation. The logarithmic relative absolute error (LRAE; Eq. (1)) is also reported at the bottom of each group of tests as well as for the global set. The label n.c. denotes non-converged calculations.

\begin{tabular}{|c|c|c|c|c|}
\hline Test & B-TCA & O-TCA & WC-TCA & $\mathrm{SOL}_{b}-\mathrm{TCA}$ \\
\hline \multicolumn{5}{|c|}{ Main group thermochemistry (kcal/mol) } \\
\hline Atomiz. energy (AE6) & 17.53 & 16.08 & 12.36 & 16.50 \\
\hline Atomiz. energy (G2/97) & 16.72 & 14.07 & 13.42 & 16.67 \\
\hline Reaction energy (BH76RC) & 3.37 & 3.25 & 5.47 & 5.97 \\
\hline Kinetics (K9) & 4.79 & 3.29 & 8.59 & 9.19 \\
\hline LRAE & -7.3 & -15.5 & -3.0 & 4.3 \\
\hline \multicolumn{5}{|c|}{ Main group geometry $\left(\mathrm{m} \AA\right.$ and $\left.\mathrm{cm}^{-1}\right)$} \\
\hline H bond lengths (MGHBL9) & 4.59 & 3.05 & 13.30 & 6.88 \\
\hline \multicolumn{5}{|c|}{ Transition metals (kcal/mol, mA, and kcal/(mol·atom) for AUnAE) } \\
\hline Atomiz. energy (TM10AE) & 10.93 & 10.17 & 12.16 & 12.22 \\
\hline Reaction energy (TMRE) & 3.16 & 4.79 & 5.49 & 6.87 \\
\hline Gold cluster AE (AUnAE) & 3.98 & 7.89 & 1.13 & 1.12 \\
\hline Bond lengths (TMBL) & 11.22 & 16.85 & 12.76 & 22.53 \\
\hline Gold clusters BL (AuBL6) & 29.76 & 32.24 & 24.51 & 27.49 \\
\hline LRAE & -2.5 & 10.7 & -8.3 & -0.4 \\
\hline \multicolumn{5}{|c|}{ Non-covalent interactions (kcal/mol) } \\
\hline Multireference AE (W4-MR) & 10.66 & 8.95 & 23.69 & 26.30 \\
\hline Difficult cases (DC9/12) & 40.94 & 44.62 & 41.01 & 48.52 \\
\hline Small interfaces (SI12) & 9.03 & 11.93 & 2.92 & 2.75 \\
\hline LRAE & -1.6 & 1.2 & -6.3 & -3.2 \\
\hline \multicolumn{5}{|c|}{ Solid-state (mA, GPa, and eV) } \\
\hline Lattice constants (LC29) & 70.92 & 73.35 & 34.46 & 34.03 \\
\hline Bulk moduli (BM29) & 14.41 & 10.66 & 8.28 & 9.70 \\
\hline Cohesive energies (CE29) & n.c. & 0.53 & 0.16 & 0.22 \\
\hline LRAE & - & 19.2 & -12.9 & -5.9 \\
\hline \multicolumn{5}{|c|}{ Overall statistics } \\
\hline Chemistry LRAE & -2.0 & 2.5 & -3.6 & -2.6 \\
\hline Solid-state LRAE & - & 19.5 & -12.6 & -5.1 \\
\hline Average LRAE & - & 10.9 & -8.3 & -4.2 \\
\hline
\end{tabular}


the accuracy in computing the surface energies or viceversa.Only few GGA functionals, specifically designed to balance the behavior in different situations (e.g. PBEint [11] or HTBS [97]), can provide some improvement over the usual behavior. Otherwise, higher level functionals need to be considered [87] (e.g the meta-GGA TPSS [21] or non-local functionals).

Inspection of the figure shows that also INT-TCA, WC-TCA, and SOL SCA can break the usual trend, providing a small, but significant, improvement with respect to most GGA functionals. In particular, $\mathrm{SOL}_{b^{-}}$ TCA yields a surface energy as accurate as PBEsol but a much better adsorption energy. Thus, it finally performs slightly better than PBEint. A similar behavior is observed for WC-TCA and INT-TCA. For these functionals we can also note that the use of the TCA correlation in place of the original ones leads to only a modest worsening of the surface energy but a significant improvement of the adsorption energy.

In the lower panel of Fig. 6] we report the interaction energy of a small molecule $\left(\mathrm{SCH}_{3}\right)$ with a metallic cluster $\left(\mathrm{Cu}_{17}\right)$. This rather simple model system constitutes a difficult test for GGA functionals, since it requires a good description of both molecular and slowly-varying systems [11]. The curves reported in Fig. [6 show that the $\mathrm{SOL}_{b}$-TCA, WC-TCA, and PBEint-TCA functionals perform well for this problem, as they give a well balanced treatment of all density regimes. Thus, they can finally outperform more specialized functionals such as PBE and PBEsol.

\section{CONCLUSIONS}

We have performed a thorough investigation of the TCA correlation functional. We have shown that, even if it does not recover the exact LDA limit, the TCA correlation performs well for solid-state problems. Consequently, the TCA functional shows a broad applicability, being accurate for a variety of systems ranging from atoms to jellium surfaces. To check the compatibility of the TCA correlation with GGA exchange functionals, we have studied its performance in conjunction with a family of PBE-like exchange functionals as well as with some other popular GGA exchange functionals. We have found that the combination of the TCA correlation with non-empirical exchange functionals gives a significant improvement in the description of molecular systems, but displays some limitations in the case of bulk solids. Nevertheless, a good accuracy and a large applicability can be achieved when the exchange part is well calibrated to balance slowly- and rapidly-varying effects appropriately.

In conclusion, the present study indicates that TCA is competitive with other state-of-the-art GGA correlation functionals, showing its usefulness for electronic structure studies. Furthermore, our assessment suggests that the WC-TCA, INT-TCA, and $\mathrm{SOL}_{b}$-TCA XC functionals are good tools for practical applications at the GGA level of theory. These functionals have shown good average accuracy and wide applicability, being above-the-average for both chemical and solid-state tests. Thus, they appear to be suitable computational tools to treat complex problems, where different density regimes are involved, e.g. etherogeneous catalysis.

\section{Acknowledgments}

We thank TURBOMOLE GmbH for providing the TURBOMOLE program package. E. Fabiano acknowledges the partial funding of this work from a CentraleSupélec visiting professorship.
[1] Hohenberg P, Kohn W (1964) Phys Rev 136:B864.

[2] Kohn W, Sham LJ (1965) Phys Rev 140:A1133.

[3] Perdew JP, Schmidt K (2001) AIP Conf Proc 577:1.

[4] Vosko SH, Wilk L, Nusair M (1980) Can J Phys 58:1200.

[5] Perdew JP, Wang Y (1992) Phys Rev B 45:13244; Perdew JP, Wang Y (1992) Phys Rev B 46:12947.

[6] Ragot S, Cortona P (2004) J Chem Phys 121:7671.

[7] Langreth DC, Perdew JP (1980) Phys Rev B 21:5469.

[8] Perdew JP, Burke K, Ernzerhof M, (1996) Phys Rev Lett $77: 3865$.

[9] Constantin LA, Fabiano E, Laricchia S, Della Sala F (2011) Phys Rev Lett 106:186406.

[10] Perdew JP, Ruzsinszky A, Csonka GI, Vydrov OA, Scuseria GE, Constantin LA, Zhou X, Burke K (2008) Phys Rev Lett 100:136406.

[11] Fabiano E, Constantin LA, Della Sala F (2010) Phys Rev B 82:113104.

[12] Constantin LA, Fabiano E, Della Sala F (2011) Phys Rev B 84:233103.

[13] Chiodo L, Constantin LA, Fabiano E, Della Sala F (2012)
Phys Rev Lett 108:126402.

[14] Becke AD (1988) Phys Rev A 38:3098.

[15] Lee C, Yang W, Parr RG (1988) Phys Rev B 37:785.

[16] Fabiano E, Trevisanutto PE, Terentjevs A, Constantin LA (2014) J Chem Theory Comput 10:2016.

[17] Tognetti V, Cortona P, Adamo C (2008) J Chem Phys 128:034101.

[18] Tognetti V, Cortona P, Adamo C (2008) Chem Phys Lett 460:536.

[19] Bremond E, Pilard D, Ciofini I, Chermette H, Adamo C, Cortona P (2012) Theor Chem Acc 131:1184.

[20] Wu Z, Cohen RE (2006) Phys Rev B 73:235116.

[21] Tao J, Perdew JP, Staroverov VN, Scuseria GE (2003) Phys Rev Lett 91:146401.

[22] Perdew JP, Ruzsinszky A, Csonka GI, Constantin LA, Sun J (2009) Phys Rev Lett 103:026403.

[23] Constantin LA, Chiodo L, Fabiano E, Bodrenko I, Della Sala F (2011) Phys Rev B 84:045126.

[24] Constantin LA, Fabiano E, Della Sala F (2012) Phys Rev B 86:035130. 
[25] Constantin LA, Fabiano E, Della Sala F (2013) J Chem Theory Comput 9:2256.

[26] Constantin LA, Fabiano E, Della Sala F (2013) Phys Rev B 88:125112.

[27] Stephens PJ, Devlin JF, Chabalowski CF, Frisch MJ (1994) J Phys Chem 98:11623.

[28] Perdew JP, Ernzerhof M, Burke K (1996) Phys Rev Lett 105:9982.

[29] Adamo C, Barone V (1999) J Chem Phys 110:6158.

[30] Cortona P (2012) J Chem Phys 136:086101.

[31] Guido CA, Brémond E, Adamo C, Cortona P (2013) J Chem Phys 138:021104.

[32] Fabiano E, Constantin LA, Della Sala F (2012) Int J Quantum Chem 13:6670.

[33] Fabiano E, Constantin LA, Cortona P, Della Sala F (2015) J Chem Theory Comput 11:122.

[34] Kümmel S, Kronik L. (2008) Rev Mod Phys 80:3.

[35] Fabiano E, Della Sala F (2007) J Chem Phys 126:214102.

[36] Grabowski I, Fabiano E, Della Sala F (2013) Phys Rev B 87:075103.

[37] Grabowski I, Fabiano E, Teale AM, miga S, Buksztel A, Della Sala F (2014) J Chem Phys 141:024113.

[38] Fabiano E, Constantin LA, Della Sala F (2012) J Chem Phys 137:194105.

[39] Zhao Y, Truhlar DG (2008) Acc Chem Res 41:157-167.

[40] Peverati R, Zhao Y, Truhlar DG (2011) J Phys Chem Lett $2: 1991$.

[41] Peverati R, Truhlar DG (2014) Phil Trans R Soc A 372:20120476.

[42] Peverati R, Truhlar DG (2012) J Chem Theory Comput 8:2310-2319.

[43] Peverati R, Zhao Y, Truhlar DG (2011) J Phys Chem Lett 2:1991.

[44] Haas P, Tran F, Blaha P, Schwarz K (2011) Phys Rev B 83: 205117.

[45] Colle R, Salvetti O (1990) J Chem Phys 93:534.

[46] Rey J, Savin A (1998) Int J Quant Chem 69:581590.

[47] Thakkar AJ, McCarthy SP (2009) J Chem Phys 131:134109.

[48] Tognetti V, Cortona P, Adamo C (2009) Theor Chem Acc 122:257.

[49] Tognetti V, Joubert L, Cortona P, Adamo C (2009) J Phys Chem A 113:12322.

[50] Tognetti V, Cortona P, Adamo C (2010) Int J Quantum Chem 110:2320.

[51] Labat F, Brémond E, Cortona P, Adamo C (2013) J Mol Model 19:2791.

[52] Tognetti V, Adamo C, Cortona P (2009) AIP Conf. Proc. 1102:147.

[53] Tognetti V, Le Floch P, Adamo C (2009) J. Comput. Chem. 31:1053.

[54] Tao J, Perdew JP, Almeida LM, Fiolhais C, Kúmmel S (2008) Phys Rev B 77:245107.

[55] Woon DE, Dunning TH (1994) J Chem Phys 100:2975.

[56] Woon DE, Dunning TH (1993) J Chem Phys 98:1358.

[57] Dunning TH (1989) J Chem Phys 90:1007.

[58] Balabanov NB, Peterson KA (2005) J Chem Phys 123:064107.

[59] Handy NC, Cohen AJ (2001) Mol Phys 99:403.

[60] Constantin LA, Terentjevs A, Della Sala F, Fabiano E (2015) Phys Rev B 91:041120(R).

[61] Lynch BJ, Truhlar DG (2003) J Phys Chem A 107:8996.

[62] Haunschild R, Klopper W (2012) Theor Chem Acc 131:1112.
[63] Karton A, Tarnopolsky A, Lamére J-F, Schatz GC, Martin JML (2008) J Phys Chem A 112:12868.

[64] Goerigk L, Grimme S (2010) J Chem Theory Comput 6:107.

[65] Goerigk L, Grimme S (2011) Phys Chem Chem Phys 13:6670.

[66] Curtiss LA, Raghavachari K, Redfern PC, Pople JA (1997) J Chem Phys 106:1063.

[67] Haunschild R, Klopper W (2012) J Chem Phys 136:164102.

[68] Zhao Y, Lynch BJ, Truhlar DG (2004) J Phys Chem A 108:2715.

[69] Zhao Y, González-Garca N, Truhlar DG (2005) J Phys Chem A 109:2012.

[70] Lynch BJ, Truhlar DG (2003) J Phys Chem A 107:3898.

[71] Zhao Y, Truhlar DG (2006) J Chem Phys 125:194101.

[72] Biczysko M, Panek P, Scalmani G, Bloino J, Barone V (2010) J Chem Theory Comput 6:2115.

[73] Furche F, Perdew JP (2006) J Chem Phys 124:044103.

[74] Bühl M, Kabrede H (2006) J Chem Theory Comput 2:1282.

[75] Fabiano E, Constantin LA, Della Sala F (2011) J Chem Phys 134:194112.

[76] Zhao Y, Truhlar DG (2005) J Chem Theory Comput 1:415.

[77] Fabiano E, Constantin LA, Della Sala F (2014) J Chem Theory Comput 10:3151.

[78] Peverati R, Truhlar DG (2012) J Chem Theory Comput 8:2310.

[79] Sun J, Marsman M, Csonka GI, Ruzsinszky A, Hao P, Kim Y-S, Kresse G, Perdew JP (2011) Phys Rev B 84:035117; Harl J, Schimka L, Kresse G (2010) Phys Rev B 81:115126; Schimka L, Harl J, Kresse G (2011) J Chem Phys 134:024116; Csonka GI, Perdew JP, Ruzsinszky A, Philipsen PHT, Lebègue S, Paier J, Vydrov OA, Ángyán JG (2009) Phys Rev B 79:155107; Janthon P, Luo S, Kozlov SM, Viñes F, Limtrakul J, Truhlar DG, Illas F (2014) J Chem Theory COmput 10:38323839.

[80] Mattsson AE, Armiento R, Paier J, Kresse G, Wills JM, Mattsson TR (2008) J Chem Phys 128:084714.

[81] TURBOMOLE, V6.3; TURBOMOLE GmbH: Karlsruhe, Germany, 2011. Available from http://www.turbomole.com (accessed May 2015).

[82] Weigend F, Furche F, Ahlrichs R (2003) J Chem Phys 119:1275312763.

[83] Weigend F, Ahlrichs R (2005) Phys Chem Chem Phys 7:3297.

[84] Dolg M, Wedig U, Stoll H, Preuss H (1987) J. Chem. Phys. 86:866.

[85] Andrae D, Häußermann U, Dolg M, Stoll H, Preuss H (1990) Theor. Chim. Acta 77:123.

[86] Kresse G, Furthmuller J (1996) Phys Rev B 54:11169.

[87] Sun J, Marsman M, Ruzsinszky A, Kresse G, Perdew JP (2011) Phys Rev B 83:121410.

[88] Wang Y, Perdew JP (1991) Phys Rev B 43:8911.

[89] Fabiano E, Constantin LA, Della Sala F (2011) J Chem Theory Comput 7:3548.

[90] del Campo JM, Gázquez JL, Trickey SB, Vela A (2012) J Chem Phys 136:104108.

[91] Clementi E, Corongiu G (1997) Int J Quant Chem 62:571.

[92] McCarthy SP, Thakkar AJ (2011) J Chem Phys 134:044102.

[93] Zupan A, Burke K, Ernzerhof M, Perdew JP (1997) J Chem Phys 106:10184. 
[94] Laricchia S, Fabiano E, Constantin LA, Della Sala F (2011) J Chem Theory Comput 7:2439.

[95] Laricchia S, Constantin LA, Fabiano E, Della Sala F (2014) J Chem Theory Comput 10:164.
[96] ONeill DP, Gill PMW (2005) 103:763.

[97] Haas P, Tran F, Blaha P, Schwarz K (2011) Phys Rev B 83:205117. 